\title{
Reposição Hormonal: vantagens e desvantagens
}

\section{Hormonal Replacement: advantages and disdvantagens}

\author{
Danieli Ribeiro Giacomini ${ }^{\text {1; }}$ Eliane Aparecida Campesatto Mella ${ }^{2}$
}

\begin{abstract}
Resumo
A deficiência de estrogênio foi considerada por muitos uma condição fisiológica ,e não patológica, provavelmente porque a insuficiência ovariana é geneticamente programada. Entretanto com o aumento da expectativa de vida das mulheres, o impacto negativo da deficiência de estrogênio tornou-se mais significativo. Embora essa deficiência seja tratável, menos de $20 \%$ das mulheres pós menopáusicas recebem estrogênio. Embora a terapia de reposição hormonal (TRH) na menopausa não seja totalmente isenta de riscos,ela tem sido utilizada com o objetivo de aliviar sintomas e de agir preventivamente e assim reduzir o aparecimento de doenças, como as cardiovasculares e a osteoporose. A terapêutica hormonal tem passado os últimos anos por duvidas e incertezas, em consequiência de diversas publicações relacionadas a seu uso, desta forma torna-se importante analisar as vantagens e desvantagens dessa terapia: analisar através de uma ampla revisão bibliográfica, as vantagens e desvantagens da terapia de reposição hormonal em mulheres climatérias, bem como avaliar se os possíveis riscos dessa terapia superam os benefícios proporcionados pela mesma. Utilizou-se como metodologia a pesquisa bibliográfica efetuada em bibliotecas e internet e os textos de livros e artigos científicos foram estudados e os conteúdos selecionados para a elaboração do trabalho.A terapia de reposição hormonal (TRH) é recomendada para alivio dos sintomas vasomotores, tratamento da atrofia vaginal e prevenção da osteoporose. Apesar das conhecidas vantagens, aproximadamente $70 \%$ das mulheres cessam o tratamento após o primeiro ano. Uma das principais causas da baixa aderência a TRH é o sangramento irregular, outras razões incluem mastalgia, náusea, cefaléia, ganho de peso e retenção hídrica, além do medo de câncer. A decisão de uma mulher em usar terapia de reposição hormonal é um processo complexo determinado pela recomendação de seu médico, pelo risco individual de doenças, pelas atitudes frente a menopausa e TRH, valores, sintomas menopausais e ao meio ao qual a mulher pertence.
\end{abstract}

Palavras-chave: Terapia de reposição hormonal, menopausa, climatério, estrogênio.

${ }^{1}$ Farmacêutica Cursando Especialização em Análises Clínicas e em Manipulação Farmacêutica

${ }^{2}$ Professora Mestre do Centro Universitário de Maringá - CESUMAR 


\begin{abstract}
The deficiency of estrogens was considered by many people as a physiological condition and not pathological one probably because the ovarian inadequacy is genetically programmed. However with the increase of the expection of the women's life, the negative impact of the deficiency of estrogens became more significant. Although this deficiency is easy to take care less than, 20\% of the women post menopáusicas receive estrogens. The therapy of hormonal replacement (THR) in the menopause it appeared as the mainly treatment form, to alleviate the symptoms and act as prevention, reducing the emergence of diseases, as the cardiovascular and the osteoporosis, but THR is not totally unproved of risks. The hormonal therapeutical has past the last years for doubts and uncertainties, in consequence of several related publications of its use, this way it becomes important to analyze the advantages and disadvantages of this therapy. Analyse througha wide bibliographical revision, the advantages and disadvantages of the TRH replacement in women climaterias, as well as evaluate if the possible risks of this therapy overcomes s the benefits proportionate for the same. It was used as methodology bibliographical researches made in libraries and internet. The texts of books and scientific articles were studied and the contents of them were selected for the elaboration of this work. The THR is recommended to alleviate the vasomotores symptoms, treatment of vaginal atrophy and prevention of osteoporosis. In spite of the acquaintances advantages, approximately $70 \%$ of the women interrupt the treatment after the first year. One of the principal causes of the low adherence of THR is the bleed irregular, other reasons includes mastalgia, nausea, chronic headache, profit of weight and hidrica retention, besides the fear of cancer. The decision of a woman to use the TRH is a complex process and it is recommended by the doctor, who is taking care of her by the individual risk of diseases, by the attitudes toward the menopause and THR, values menopausais, symptoms and to the middle to which the woman belongs.
\end{abstract}

Key words: Therapy of hormonal replacement. Menopause. Climacteric. Estrogens.

\section{Definição}

A deficiência de estrogênio foi considerada por muitos uma condição fisiológica, e não patológica, provavelmente porque a insuficiência ovariana é geneticamente programada. Entretanto com o aumento da expectativa de vida das mulheres, $o$ impacto negativo da deficiência de estrogênio tornou-se mais significativo.

Embora essa deficiência seja tratável, menos de $20 \%$ das mulheres pós menopáusicas recebem estrogênio. A terapia de reposição hormonal (TRH) embora não totalmente desprovida de riscos, surgiu com o propósito de aliviar sintomas e de agir preventivamente, e reduzir assim o aparecimento de doenças, como as cardiovasculares e a osteoporose.

A decisão de uma mulher em usar essa terapia é um processo complexo determinado pela recomendação de seu médico, o risco individual de doenças, as atitudes frente à menopausa, aos valores, sintomas menopausais e ao meio ao qual a mulher pertence.

A terapêutica hormonal tem passado, nos últimos anos por dúvidas e incertezas, em conseqüência de diversas publicações relacionadas a seu uso e desta forma, torna-se importante analisar as vantagens e desvantagens dessa terapia, uma vez que surge de tempos em tempos, uma literatura contraditória a esse respeito.

\section{Desenvolvimento}

\section{Climatério}

Compreende-se como climatério a fase da vida na qual as gônadas femininas ( ou masculinas) cessam sua função e a ocorrência do último sangramento cíclico é designado, a menopausa. O período precedente, em que a menstruação já se apresenta irregular, é identificado como pré-menopausa e o período subseqüente á menopausa, no qual dominam 
as manifestações regressivas, como pós-menopausa (FERREIRA, 1999).

O climatério tem inicio com o cessar da função reprodutiva e termina com a adaptação do organismo á ausência de gônadas. Quando se esgota a reserva de folículos ovarianos em todos os estágios de maturação, sua função declina progressivamente. Isso ocorre, via de regra, ao redor dos 45 anos. A duração desta fase é muito variável, pode abranger algumas semanas, como também muito lenta de 10 a 20 anos. As diversas fases do climatério caracterizam-se por um comportamento diverso das glândulas endócrinas (DOUGLAS, 2002).

O período que precede a menopausa é caracterizado por um grau variável de alterações somáticas que refletem alterações no funcionamento normal do ovário. Em algumas mulheres, a irregularidade menstrual é o sintoma mais importante da transição para a menopausa. Como o sangramento anormal é um dos sintomas mais comuns de problemas uterinos, a irregularidade menstrual durante a perimenopausa deve ser avaliada cuidadosamente. Frequentemente, o sangramento uterino associado a este período de transição é secundário a flutuações fisiológicas normais do estrogênio, e não a alguma patologia subjacente, e pode ser tratado clinicamente (HURD; AMESSE; RANDOLPH, 2005).

Na pré-menopausa, cessa a função reprodutiva, desaparecem as ovulações regulares e a formação de corpo lúteo, uma vez que a reserva de folículos suscetíveis de maturação encontra-se esgotada. Os estrogênios continuam sendo secretados em quantidades variáveis, portanto, a função vegetativa do ovário é mantida (ALDRINGHI; FALUDI; MANSUR, 2005).

A menopausa está associada à interrupção da menstruação resultante da perda da função folicular ovariana, e deve ser caracterizada como um evento e não como um período. O momento da menopausa é determinado geneticamente e ocorre em uma idade média de 51 anos (2-4). Não está relacionado à raça nem ao estado nutricional. No entanto verificase que a menopausa ocorre mais cedo em mulheres nulíparas, em tabagistas e em algumas mulheres submetidas à histerectomia (BARACAT et al., 2005).

Tradicionalmente, a menopausa tem sido diagnosticada retrospectivamente com base na ausência de períodos menstruais. Com o advento dos modernos exames laboratoriais, agora a menopausa pode ser definida mais precisamente como amenorréia, com sinais de hipoestrogenemia, elevado nível sérico de hormônio folículo-estimulante (FSH), acima de 40 UI/ml (HAIDAR; BOUNDUKI; BARACAT, 1999).

A menopausa também pode ser diagnosticada com base em sintomas subjetivos, como fogachos, ou com base em estudo de estímulos, como o teste de supressão com progesterona. Fogachos e outros sintomas agudos associados ao período da perimenopausa frequentemente se tornam mais intensos próximos da menopausa, quando os níveis de estrogênio circulante caem subitamente. Estes sintomas são particularmente intensos em paciente com insuficiência ovariana prematura ou menopausa cirúrgica e são acompanhados por redução graduais dos estrogênios circulantes (HURD; AMESSE; RANDOLPH, 2005).

O período pós-menopausa é de relativa quiescência ovariana após a menopausa. Durante este período prolongado, as mulheres são vulneráveis a distúrbios causados por deficiência de estrógeno. Embora longo prazo, o impacto da deficiência de estrogênio sobre a saúde possa ser semelhante ao dos distúrbios tireoidianos ou suprarenais, até recentemente foi dada relativamente pouca atenção a este problema. Por essa razão, a terapia de reposição hormonal é uma das principais preocupações na assistência a saúde de muitas mulheres na pós-menopausa (CHUERY et al., 2005). 


\section{Preocupações da Paciente com a Menopausa}

Frequentemente, há um amplo espectro de emoções associado às alterações hormonais e corporais características deste período. A resposta de uma paciente à menopausa pode ser afetada por fatores como estilo de vida e o controle do processo de envelhecimento. A perda da fertilidade e da função menstrual que acompanha a menopausa natural ou cirúrgica pode ter impacto sobre a sensação de bem estar de uma mulher. O médico deve ser sensível ao sofrimento emocional, que pode ser significativo, enfrentado por mulheres que entram na menopausa, e deve estar preparado para oferecer apoio psicológico. Sinais sutis da transição para a menopausa podem se negligenciados, porque a mulher pode hesitar em relatar sintomas incomuns e, possivelmente, relacionados aos hormônios. Se não for realizado um questionamento direto, esses sintomas frequentemente não serão detectados, e isso resultará em atraso no diagnóstico e no tratamento (HURD; AMESSE; RANDOLPH, 2005).

\section{Repercussões do Climatério}

Dentre as alterações que o hipoestrogenismo pode determinar na mulher, assinalam-se às ginecológicas e as extras ginecológicas.Salientamse, dentre os sintomas ginecológicos as disfunções menstruais e urogenitais e as alterações da genitália interna e externa. As modificações nãoginecológicas estão representadas pelos sintomas vasomotores, neuropsíquicos, as alterações atróficas da pele e anexos, atrofia das mucosas nasais e oculares, diminuição da acuidade auditiva, gengivite e descalcificação dos dentes, comprometimento do SNC ( principalmente a doença de Alzheimer) e alterações do metabolismo ósseo e cardiovascular (BARACAT et al., 2005).

\section{Sangramento Uterino}

O padrão menstrual reflete o equilíbrio hormonal esteroídico. Na perimenopausa, a maioria das mulheres experimentam irregularidades de seus ciclos, decorrentes não só da redução da freqüência ovulatória, mas também das concentrações erráticas dos hormônios ovarianos. Aproximadamente 90\% das mulheres manifestam sangramento uterino irregular de quatro a oito anos antes da menopausa. (GONÇALVES, et al., 2005).

Segundo Wall, Dona e Okuda (2003), diferentemente do sangramento irregular, há o sangramento uterino anormal, que se caracteriza por quantidade volumosa ou duração maior que sete dias, ou intervalos inferiores há 21 dias, nesses casos, é necessário uma investigação apurada. No diagnóstico diferencial deve ser considerado o uso inadequado de contraceptivos orais, a presença de miomas, pólipos, hiperplasias endometriais, câncer do colo e/ou endométrio, hipotireodismo e discrasias sanguíneas.

\section{Ondas de Calor e Suores Noturnos}

Ondas de calor e suores ocorrem de repente, em geral na parte superior do tórax, pescoço e rosto. Às vezes, essas ondas são desencadeadas por uma situação estressante, uma bebida quente e podem ocorrer de modo ocasional e repetirem-se várias vezes ao dia mas não constituírem motivo para preocupação (POLDEN; MANTLE, 1998).

Os fogachos ou ondas de calor representam o segundo sintoma mais frequiente da perimenopausa (58\%), só perdendo para as irregularidades menstruais, são considerados "marca registrada" da deficiência estrogênica, apesar de ocorrerem em outras situações (hipertiroidismo, leucemia, carcinóides, feocromocitoma e tumores pancreáticos). Potencialmente podem alterar a qualidade de vida, seja na atividade cotidiana, seja na qualidade do sono. A etiologia dos fogachos é motivo de muita especulação, embora uma possível causa seja a redução dos estrogênios sobre os neurotransmissores cerebrais (VIGETA; BRETAS, 2004).

Classicamente, as ondas de calor acontecem á noite e consequentemente, tendem a afetar a qualidade do sono, promovendo distúrbios 
psíquicos como fadiga e irritabilidade, além da diminuição do poder de concentração e da memória (DOUGLAS, 2002).

\section{Alterações Urogenitais e Mamárias}

As alterações urogenitais ocorrem em 1/3 das mulheres acima dos 50 anos e decorrem do hipoestrogenismo, que promove atrofia do epitélio vaginal, decréscimo da elasticidade e perda da rugosidade, diminui também o fluxo sanguíneo vaginal, e ocasiona perda da habilidade de lubrificação em resposta a estimulação sexual. Com isso, prurido e irritação locais se tornam freqüientes. Todos esses efeitos têm menor probabilidade de ocorrerem em mulheres que possuam vida sexual regular. Outra manifestação, a incontinência urinária afeta 10 a $30 \%$ das mulheres entre 50 e 64 anos; sua relação com a menopausa não tem sido comprovada. A associação entre incontinência uretral e esteróides sexuais, decorre da presença de receptores de estrogênios na uretra e no colo da bexiga, que possuem a mesma origem embriológica que a vagina, além do mais, há casos de incontinência urinária que melhoram com a estrogenoterapia (ORCESI, et al., 2003).

O hipoestrogenismo leva a hipotrofia das mamas e do aparelho urogenital:as mamas diminuem de tamanho e tornam-se flácidas, havendo substituição gradual do parênquima por gordura. Os tecidos do aparelho urogenital, compostos de epitélios de revestimento, ligamentos e camadas musculares, perdem o turgor, e tornam-se mais adelgaçados (SANTOS; PESSOLI; IOSHII, 2001).

\section{Alterações Cutâneas}

Sabe-se que o conteúdo de colágeno da pele é dependente de estrógeno. A perda de colágeno resulta em que o mesmo se torna mais delgado e menos elástico. Os receptores de estrógeno foram encontrados nas glândulas sebáceas, e isso talvez seja responsável pela secura da pele de que certas mulheres se queixam eé anunciada pelos fabricantes de cosméticos (PIATO, 1997).
Estudos mostram significativa relação entre o declínio do colágeno cutâneo e os anos de pósmenopausa. Assim, é muito mais rápido nos primeiros anos após a menopausa (perda de $30 \%$ de colágeno cutâneo nos primeiros cinco anos). Por outro lado, nenhuma correlação tem sido encontrada entre conteúdo de colágeno e idade cronológica, reforçando as evidencias de que os anos de pósmenopausa são mais importantes do que a idade. As conseqüências clinicas da menopausa sobre a pele incluem enrugamento, secura e adelgaçamento, tornado-a mais susceptível a traumas. Tanto a exposição solar como o tabagismo pode potencializar os efeitos da menopausa sobre a pele (WALL; DONA; OKUDA, 2003).

\section{Disfunção Sexual}

Uma grande preocupação de algumas mulheres é a diminuição da libido ou da satisfação sexual que pode ocorrer com a menopausa natural ou cirúrgica. No entanto, a atividade sexual permanece relativamente estável em mulheres na menopausa. Apesar disso, apenas metade das mulheres neste período relata atividade sexual. Isso pode estar relacionado a uma diminuição do número de parceiros disponíveis em uma população idosa (HURD; AMESSE; RANDOLPH, 2005).

Não se sabe que intensidade a menopausa altera a função sexual. No entanto, está bem claro que o declínio dos estrogênios séricos não se associa com a redução da libido. Há estudos demonstrando que alterações no desejo sexual podem decorrer do impacto negativo do hipoestrogenismo sobre o fluxo sanguíneo arterial e sobre o Sistema Nervoso Central e isso agravaria a percepção ao tato e a excitação. A libido nas mulheres, por sua vez, se associa aos androgênios, principalmente à testosterona (DOUGLAS, 2002).

O papel dos androgênios na libido antes e após a menopausa é incerto. Embora tenha sido descrito que os níveis de testosterona são menores em mulheres na pós do que na pré-menopausa, as concentrações circulantes não se modificam na 
menopausa, e os níveis de testosterona livre, poderiam, na verdade, aumentar durante alguns meses, antes de diminuírem até atingirem níveis menores que os observados em mulheres na prémenopausa. Em contraste, há uma redução acentuada dos níveis de androgênios após a ooforectomia. Em homens, a relação entre androgênios e libido foi bem estabelecida. Por essa razão, alguns clínicos defenderam o tratamento com androgênios em mulheres que apresentam diminuição da libido. Entretanto, nas mulheres não foi demonstrado que a diminuição dos androgênios circulantes após a menopausa altera regularmente a libido. As evidencias disponíveis sugerem que a satisfação sexual em mulheres na pós-menopausa não diminui com o passar do tempo, tornando controverso o tratamento com androgênios (MARINHO et al., 2005).

\section{Alterações do Humor e Comportamento}

Diferentemente do que se propaga, não há evidencias cientificas de que a menopausa seja responsável direta pela eclosão da depressão. Entretanto, pacientes na perimenopausa referem maior cansaço, irritação e depressão, o que poderia ser explicado pelas flutuações do estrogênio, que causam impacto negativo sobre a atividade dos neurotransmissores cerebrais. É importante ressaltar que quadros depressivos na perimenopausa e, mesmo após a menopausa,podem decorrer de ondas de calor não tratadas, que ao gerarem sono de má qualidade propiciam irritabilidade, perda da sensação de bem-estar, e distúrbios cognitivos (ALDRINGHI; FALUDI; MANSUR, 2005).

Entretanto, múltiplos estudos não verificaram sinais sugestivos de que os sintomas psicológicos apresentados durante a transição para a menopausa estivessem relacionados com as alterações do estrogênio. $\mathrm{O}$ aumento da ansiedade e da irritabilidade associado a fatores psicossociais do que ao estado estrogênico. É importante não apenas investigar e tratar o conjunto de sintomas associados átransição para a menopausa, mas também perceber que a intervenção psicológica pode ser útil para algumas mulheres (FRANÇA; ALDRIGHI; MARUCCI, 2005).

\section{Sistema Nervoso Central}

A deficiência de estrogênioparece ter efeitos sobre o sistema nervoso central, os quais somente recentemente foram determinados. As mulheres na perimenopausa frequentemente têm dificuldade de concentração e perda de memória de curto prazo. Estes sintomas foram atribuídos aos efeitos do envelhecimento isolado ou da sutil privação do sono associado a fogachos. O estrogênio parece ter efeitos diretos sobre a função mental. O estrogênio parece ter efeitos diretos sobre a função mental, e a terapia de reposição mostrou melhorar a memória de curto e de longo prazo em mulheres na pós-menopausa (ALDRINGHI; FALUDI; MANSUR, 2005).

Muitos são os fatores envolvidos na função cognitiva (concentração e memória). Entre eles salientam-se, o estilo de vida e a condição social. Os hormônios, por sua vez, tem sido objeto de intensa investigação, pois áreas do cérebro envolvidas na cognição (córtex, lobos temporais e sistema límbico) contem expressivas quantidades de receptores de estrogênios,o o que explica, as dificuldades na memória recente e da concentração em mulheres na perimenopausa (CLAUPAUCH et al., 2002).

\section{Doença Cardiovascular}

A doença cardiovascular (DCV) tem sido associada a múltiplas causas, a mais importante das quais pode ser a idade. $\mathrm{O}$ risco de doença cardiovascular aumenta nos homens e nas mulheres durante toda a vida. Embora o risco de morte por doença coronariana seja pelo menos três vezes maior nos homens que nas mulheres antes da menopausa, o risco relativo aumenta significativamente após a menopausa. É essencial estar ciente dos fatores de risco evitáveis para a doença cardiovascular e incentivar as mulheres a minimizar estes fatores de riscos (FRANÇA; ALDRIGHI; MARUCCI, 2005). 
A DCV é incomum na pré-menopausa. Quando a mulher atinge a menopausa, o risco aumenta, sugerindo que o estrogênio exerça benefícios cardioprotetores. Inúmeras explicações têm sido relatadas para explicar o porquê de a redução do estrogênio aumenta o risco da DCV. Ao examinar as possíveis razoes do aumento da DCV em mulheres após a menopausa verifica-se que o achado mais prevalente são as alterações sobre as lipoproteínas, ou seja, a elevação significativa do colesterol LDL a redução do colesterol HDL, o que propicia um perfil proaterogenico (WALL; DONA; OKUDA, 2003).

Em um grande estudo, verificou-se que mulheres que nunca haviam utilizado estrogênios o aumento do colesterol LDL após a menopausa foi de $4 \%$ em relação aos valores observados na prémenopausa. Os efeitos diretos sobre os vasos que ocorrem após a menopausa são considerados, quanto ao risco do DCV mais importantes do que as alterações lipoprotéicas. A menopausa não altera o equilíbrio da coagulação; de fato, fatores fibrinoliticos, como a antitrombina 3 e o plasminogenio, aumentam paralelamente com os fatores pró-coagulantes (fator VII e fibrinogênio). Após a menopausa, o fluxo sanguineo reduz-se em todos os leitos vasculares, bem como as concentrações séricas de prostaciclina (ALDRIGHI; FALUDI; MANSUR, 2005).

\section{Osteoporose}

A associação entre a menopausa natural e a cirúrgica e a osteoporose foi claramente estabelecida. Por definição, a osteoporose é a redução da quantidade de osso. Como esta definição, pode ser muito ampla para ser clinicamente útil, por isso alguns autores estreitaram a definição para incluir apenas a perda óssea que evolui até o ponto, em que partes específicas do esqueleto se tornem tão finas e passem a ter maior suscetibilidade a fraturas ou em que realmente há fraturas. O grau de perda óssea cortical e trabecular necessário para atender a esse critério são incertos em quem ainda não tiver uma fratura óssea. $\mathrm{O}$ numero de mulheres idosas que sofrem fraturas vertebrais por esmagamento ou fraturas de radio ou do colo do fêmur relacionado á osteoporose tem atingido proporções epidêmicas (HURD; AMESSE; RANDOLPH, 2005).

Na menopausa, aumenta a renovação e diminui a formação óssea em cada unidade de remodelação, o que conduz a uma perda de massa óssea. O risco de osteoporose depende tanto da massa óssea máxima alcançada nos anos da idade adulta jovem quanto do índice de perda da massa nas épocas posteriores. O pico de massa óssea geralmente não e alcançados antes de 30 anos e o estilo de vida é um importante determinante da probabilidade de desenvolver mais tarde osteoporose. Dentre os fatores de risco está a ausência de atividade regular e de terapia de reposição hormonal, bem como fatores genéticos e os relativos a dieta. Não se sabe com certeza em que idade começa a perda óssea, mas acredita-se que, entre 40 anos e menopausa, as mulheres perdem aproximadamente $0,3 \%$ a $0,5 \%$ de sua massa de osso cortical por ano, após a menopausa, este ritmo acelera para $2 \%$ a $3 \%$ ao ano (LANZILLOTTI et al., 2003).

Na menopausa, a principal alteração biológica é o cessar da ovulação, confirmada quando a menstruação se interrompe por pelo menos um ano. Após a menopausa, os ovários tornam-se inativos e ocorre mínima ou nenhuma liberação de estrogênio, coincidindo com a redução da absorção de cálcio pelo intestino, devido a baixa produção de calcitonina, hormônio que inibe a desmineralização óssea. O déficit de estrogênio é um determinante importante na perda óssea durante a menopausa e, quando precoce, o risco é muito maior (HARDY; OSIS; MARTINEZ, 2002).

\section{Avaliação clínico-ginecológica no climatério}

Deve-se basear no quadro clínico, pois a amenorréia com duração de pelo menos um ano, após os 40 anos, principalmente se acompanhada 
de sintomas vasomotores, é bastante sugestiva. Podem-se confirmar laboratorialmente, pelos elevados valores de FSH (acima de $40 \mathrm{mUI} / \mathrm{ml}$ ). Sintomas vasomotores e ou irritabilidade nervosa, sem causa aparente, em pacientes na prémenopausa, as quais menstruam ainda razoavelmente bem, pode indicar a necessidade de terapêutica hormonal. Nesses casos, pode-se dosar o FSH nos primeiros dias do ciclo e sete a 10 dias depois, valores elevados indicam hipoestrogenismo relativo (insuficiência ovariana). Outro método propedêutico consiste na administração de citrato de clomifeno ( $50 \mathrm{mg}$, do $5^{\circ}$ ao $9^{\circ}$ dia), dosa-se FSH na véspera e entre os dias $9 \mathrm{e} 11$. Valores acima de $26 \mathrm{mUI} / \mathrm{ml}$ sugerem haver menor reserva folicular. Na vigência de amenorréia mais prolongada, além do FSH e LH elevados, o estradiol encontra-se baixo (MARINHO et al., 2005).

Rotineiramente não é costume dosar hormônios em face do quadro clinico, quando necessário, apenas o FSH porque o teor de estradiol flutua bastante, consoante o grau de transformação periférica de androgênios e os níveis de SHBG (globulina ligadora de hormônios sexuais). Melhor é mais elucidativo do que a dosagem isolada de estradiol éo teste o provocativo de progestagênios ou teste de progesterona em mulheres com amenorréia. Se negativos, os valores indicam que os níveis de estrogênios estão baixos, aquém dos valores encontrados na fase proliferativa tardia. $\mathrm{Na}$ duvida pode-se fazer o teste medicamentoso, com pequenas doses de estrogênios (estrogênios conjugados $-0,3$ ou $0,625 \mathrm{mg}$ ) por 15 a 20 dias. Se houver melhora sintomatológica, confirma-se o diagnostico (SOCIEDADE BRASILEIRA DE CLIMATÉRIO, 2005).

Conclui-se, pois, que o diagnóstico é, sobretudo clínico havendo poucas vezes a necessidade de avaliação hormonal. Contudo, na menopausa dita precoces, é necessária maior investigação para saber se a amenorréia decorre mesmo de falência ovariana ou se ela é devida a outras causas, como hiperprolactinemia, hipotireodismo, tumores hipotalâmicos, doenças auto-imunes, entre outras. Antes de se fazer a terapia hormonal, exigem-se exames subsidiários: colposcopia, colpocitologia oncológica, mamografia, ultra-sonografia pélvica e teste provocativo com progestagênios, hemograma, glicemia, fosfatase alcalina, colesterol total e frações (HDL e LDL), triglicérides, cálcio e fósforo plasmáticos, calciúria de 24 horas e densitometria óssea. Se impossível, fazer pelo menos a citologia, o teste provocativo de progestagênios e a mamografia. Não se deve, de modo algum, começar a terapia hormonal sem o teste de progestagênio e a mamografia (MARINHO et al., 2004).

\section{Terapia de reposição hormonal (TRH)}

A deficiência de estrogênio foi considerada por muitos um distúrbio fisiológico, e não patológico, provavelmente porque a insuficiência ovariana é genéticamente programada. Entretanto, com o aumento da expectativa de vida das mulheres, o impacto negativo da deficiência prolongada de estrogênio torna-se mais significativo. Embora a deficiência de estrogênio seja tratável, menos de 20 $\%$ das mulheres na pós-menopausa tomam estrogênio (HURD; AMESSE; RANDOLPH, 2005).

O declínio da secreção de estrogênio pelos ovários é um processo lento e gradativo, que se estende por alguns anos depois que as menstruações desaparecem. É comum observar que os sintomas da menopausa são mais graves depois da interrupção súbita dos estrogênios (p. ex., depois da ooforectomia) que com a menopausa natural. No tratamento das mulheres na pós-menopausa com estrogênios, dois aspectos muito importantes são as profilaxias da perda óssea e a atenuação dos sintomas vasomotores, que são efeitos benéficos comprovados deste tratamento (STANCEL; MITCHEL; LOOX, 2003).

Nas décadas de 1960 e 1970, houve um amento da utilização da terapia de reposição estrogênica (apenas estrogênio) em mulheres na pós-menopausa principalmente para reduzir os sintomas vasomotores, a vaginite e a osteoporose. No inicio 
da década de 1980, estudos epidemiológicos indicaram que esse tratamento estava associado a um aumento significativo da incidência de carcinoma de endométrio, o que, pelo menos em parte, poderia ser atribuído á estimulação continua da hiperplasia endometrial pelos estrogênios atuando sem oposição. Essa descoberta resultou na utilização da terapia de reposição hormonal, que inclui tanto um estrogênio por seus efeitos benéficos, quanto um progestogênio para limitar a hiperplasia do endométrio (CAMPIOLO; MEDEIROS, 2003).

Embora as ações da progesterona no endométrio sejam complexas, seus efeitos na hiperplasia induzida pelo estrogênio podem envolver a redução da quantidade de receptores para estrogênios; o aumento da conversão local do estradiol em estrona menos potente via indução da 17 betahidroxisteróides desidrogenase nos tecidos; e/ou conversão do endométrio do estado proliferativo ao secretor. Atualmente, a terapia de reposição hormonal com estrogênio e progestogênio é recomendada para as mulheres que já passaram pela menopausa e têm útero. Para as mulheres com útero que não conseguem tolerar os progestogênios, ou que apresentam risco elevado de doença cardiovascular devido aos perfis desfavoráveis das lipoproteínas, o uso isolado dos estrogênios tornase preferível. Para as pacientes submetidas a histerectomia, o carcinoma de endométrio deixa de ser preocupante e o estrogênio costuma ser mais usado isoladamente, tendo em vista os efeitos deletérios potencialmente associados aos progestogênios (SANTOS; PESSOLE; IOSHII, 2001).

A terapia de reposição hormonal da menopausa (TRHM) tem sido empregada de forma crescente, visando a benefícios a curto, médio e longo prazo. Ogrande interesse em evidenciar os potenciais riscos e benefícios exercidos pela TRHM, e proporcionar melhor qualidade de vida as mulheres menopausadas tem levado á busca de respostas concretas aos potenciais benefícios e riscos desta medida, por meio de diferentes estudos epidemiológicos e ensaios clínicos (CAMPIOLO; MEDEIROS, 2003).

\section{Indicações e contra-indicações da TRH}

A terapia hormonal no climatério tem indicações precisas, dependo da fase em que a mulher se encontra, isto é, na pré, peri e pós-menopausa. $\mathrm{Na}$ pré e perimenopausa, a terapia hormonal é recomendada para corrigir as disfunções menstruais decorrentes dos distúrbios da fase lútea ou dos ciclos anovulatórios. Preconiza-se tal tratamento, também, para debelar os sintomas vasomotores que possivelmente ocorram nesse período. Na pósmenopausa, a hormonioterapia é utilizada para eliminar ou aliviar os sintomas e sinais decorrentes do hipoestrogenismo, como sintomas vasomotores e neuropsíquicos, atrofia urogenital, distúrbios urinários, alterações da pele e das mucosas, sintomas osteoarticulares, disfunção sexual, entre outros. Também tem grande valia na prevençãoe até mesmo na terapêutica da osteoporose. A terapia hormonal é indicada, ainda, nos casos em que há alterações atróficas das mucosas oral e nasal, da conjuntiva ocular e do aparelho auditivo (CHUERY et al., 2005).

Segundo VIGETA, BRETAS , 2004 constituem contra-indicação absolutas aos estrogênios:

- Neoplasias malignas de mama e endométrio recentes, dependendo do estágio clinico;

- Hepatopatia severa ativa.

- Tromboembolismo agudo.

- Sangramento genital anormal de causa desconhecida.

- Porfiria.

Quanto às contra-indicações relativas aos estrogênios, assinalam-se:

- Tromboembolismo venoso prévio.

- Doença coronariana estabelecida.

- Hipertensão arterial severa ou hipertensão previa sem estabilização após tratamento. 
- Diabete melito, não controlada.

- Historia familiar de câncer de endométrio e mama.

- Antecedentes de hiperplasia atípica de mama.

- Doenças auto-imunes em atividade (lupus eritematoso sistêmico).

- Meningioma.

- Mioma uterino e endometriose pregressa.

- Doença da vesícula biliar.

- Melanoma.

\section{Hormônios e esquema de reposição}

A base da reposição hormonal na ausência de função ovariana é o estrogênio, geralmente com o acréscimo de um progestogênio. A terapia de reposição padrão consistia na administração concomitante de estrogênio e progestogênio a qualquer paciente com o útero intacto. Mulheres submetidas a histerectomia recebiam apenas estrogênio. A melhor compreensão dos efeitos dos hormônios no corpo alterou estas condutas clássicas de tratamento. Os hormônios utilizados na TRH são: estrogênio, estrogênio mais progesterona cíclica, estrogênio mais progesterona contínua, moduladores seletivos do receptor de estrogênio e testosterona (AIDRINGHI; FALUDI; MANSUR, 2005).

Nas décadas de 1960 e 1970, houve um aumento da utilização da terapia de reposição estrogênica (i. e., apenas estrogênio) em mulheres na pós-menopausa principalmente para reduzir os sintomas vasomotores, a vaginite e a osteoporose. No inicío da década de 1980, estudos epidemiológicos indicaram que esse tratamento estava associado a um aumento significativo da incidência de carcinoma de endométrio, provavelmente devido em parte à estimulação contínua da hirperplasia endometrial pelos estrogênios atuando sem oposição. Essa descoberta resultou na utilização da terapia de reposição hormonal, que inclui tanto um estrogênio por seus efeitos benéficos, quanto um progestogênio para limitar a hiperplasia do endométrio (STANCEL; MITHEL; LOOX 2003).

Os regimes terapêuticos podem ser administrados de maneira cíclica ou contínua, incluindo autilização de estrogênios isolados, progestógenos isolados, combinações de ambos e outros hormônios. Os esquemas terapêuticos mais utilizados são: estrogênio isolado cíclico ou contínuo, progestógenoisolado cíclico ou contínuo, estrogênio cíclico e progestógeno cíclico, estrogênio contínuo e progestogênio cíclico, estrogênio contínuo e progestógeno cíclico trimestral ou quadrimestral, estrogênio e progestógenio combinados contínuos, estrogênio e androgênio contínuos ou cíclicos, estrogênio e androgênio contínuos e progestógenos cíclicos, tibilonacontínua(SOCIEDADEBRASILEIRA DECLIMATÉRIO, 2005).

Dentre os tipos de estrogênio, os que podem ser utilizados para a, TRH são: 17 -beta estradiol, estrogênios conjugados, estriol, promestriene, benzoato de estradiol, valerato de estradiol e estradiol. A estrona é duas vezes menos potente que o estradiol e possui forte ação estrogênica. $\mathrm{O}$ estradiol é dez vezes mais ativo que o estriol, pois possui maior afinidade e dissociação mais lenta com o receptor. $\mathrm{O}$ etilinilestradiol é o sintético mais potente, pois possui absorção intestinal elevada e pequena metabolização na primeira passagem pelo fígado. Os tipos de progestogênos utilizados para a reposição hormonal são; acetato de medroxiprogesterona, noretindrona, acetato de noretindrona, nomegestrol, levonorgestrel, noretisterona, acetato de ciproterona e acetato de noretisterona (CHUERY et al., 2005).

Comparando a via oral com a transdérmica, a primeira necessita maiores doses de hormônios, pois os mesmos são metabolizados na parede do trato gastrintestinal e na sua primeira passagem pelo fígado. Isto resulta em perda de aproximadamente $30 \%$ de sua atividade e estimulo de proteínas e enzimas hepáticas. Além disso, a terapia oral produz efeito maior no aumento do HDL, diminuição do LDL, aumento nos triglicérideos, e estimula a produção de angiotensinogênio (substrato de renina), 
que pode elevar a pressão arterial, e de proteínas transportadoras de hormônios sexuais. A via transdérmica necessita doses menores que a terapia oral, pois sua absorção é alta e não sofre inativação hepática. A terapia vaginal é mais apropriada para mulheres com sintomas vulvovaginais ou urinários. Ocorre absorção sistêmica em níveis um quarto daqueles alcançados com dose oral semelhante (HURD; AMESSE; RANDOLPH, 2005).

O progestogênio contínuo é utilizado nas mulheres que possuem contra-indicações a terapia estrogênica. Nas pacientes que estão na perimenopausa com alterações menstruais, emprega-se progestogênio cíclico por 10 a 14 dias por mês. Com o estabelecimento da menopausa, utiliza-se outro esquema de TRH. Ainda nas pacientes na perimenopausa com sintomas dependentes de estrogênio, o esquema combinado cíclico pode ser utilizado. O sangramento por privação hormonal ocorre logo após o termino do progestogênio. Utiliza-se estrogênio por 21 a 28 dias por mês e progestogênio por 10 dias por mês. O tempo de utilização do progestogênio é importante na redução da incidência de hiperplasia endometrial, essa porcentagem em $4 \%$ nas que utilizam progestógeno por 7 dias, para $2 \%$ nas que usam por 10 dias, e quase desaparecendo nas que usam por 12 dias, na apuração desses dados respeitaram-se as variações decorrentes das diferenças de absorção entre pacientes ou na sensibilidade endometrial (MARINHO et al., 2005).

\section{Vantagens e Desvantagens da Terapia de} Reposição Hormonal

De acordo com Rang; Dale e Ritter, (2001) ,os riscos da estrogenoterapia parecem estar relacionados á dose. Muitos dos efeitos colaterais dos contraceptivos orais em alta dosagem não ocorreram com as menores doses de estrogênio usadas para a terapia de reposição hormonal. Devido à sensibilidade de alguns tecidos ao estrogênio, devem ser considerados os possíveis riscos de estrogênio em mulheres na pósmenopausa. O uso dos estrogênios na TRH apresenta algumas desvantagens, que são as seguintes: aumento no risco de câncer de endométrio; possível aumento no risco de câncer de mama; sangramento uterino; sintomas gastrintestinais menores e alterações do humor e pequeno aumento no risco de tromboembolismo venoso e embolia pulmonar.

A terapia de reposição hormonal (TRH) é recomendada para o alívio dos sintomas vasomotores, tratamento da atrofia vaginal e prevenção da osteoporose. Apesar das conhecidas vantagens, aproximadamente $70 \%$ das mulheres cessam o tratamento após o primeiro ano. Uma das principais causas da baixa aderência a TRH é o sangramento irregular, outras razões incluem mastalgia, náusea, cefaléia, ganho de peso e retenção hídrica, além do medo de câncer de mama (NAHÂS et al., 2003).

Segundo Campiolo e Medeiros (2003), recentes estudos observacionais têm indicado possível aumento no risco de tromboembolismo venoso com a TRH. Essas observações levaram á suspeita de que estrogênios exógenos, como os usados na TRH, poderiam elevar o risco de tromboembolismo. Esse risco de tromboembolismo com a TRH parece ser bem maior em mulheres com história pessoal ou familiar de trombose venosa profunda ou embolismo pulmonar, assim como presença de severas veias varicosas, obesidade, cirurgia, trauma, câncer, ou permanência no leito por longo período.

\section{Vantagens da TRH em Relação à Doença Coronária Isquêmica}

O estrogênio exerce diversas ações benéficas sobre os parâmetros de risco cardiovascular em estudos clínicos. Os principais efeitos são: melhora do perfil lipidico, do fluxo vascular arterial avaliado por dopplervelocimetria em varias artérias, da pressão arterial e do debito cardíaco. Dados experimentais relatam, entre outros benefícios, efeitos vasodilatadores nas artérias devido à liberação de oxido nítrico pelo endotélio e outras substâncias que controlam o tônus arterial. Estudos 
mostram ação antioxidante do estrogênio na placa ateromatosa e diminuição da resistência periférica á insulina (MARINHO et al., 2005).

Existem diversos mecanismos pelos quais os estrogênios poderiam proteger o coração. Os mecanismos pelos quais o estrogênio poderia proteger o coração o mais importante parece ser o efeito do estrogênio sobre os lipídios e lipropoteínas séricas. O estrogênio diminui os níveis circulantes de lipoproteínas de baixa densidade e aumenta os níveis de lipoproteínas de alta densidade. Tanto a diminuição absoluta do colesterol total, quanto o aumento da razão entre lipoproteína de alta densidade e de baixa densidade parecem retardar a progressão da doença coronariana. Embora estes efeitos sejam atenuados pelo uso concomitante de progestogênio, este efeito parece diminuir com o tempo. Foi estimado que o estrogênio é responsável por 20 a $50 \%$ do efeito protetor total contra doença cardiovascular. Não se conhece o grau em que os progestogênios podem diminuir este efeito, se é que diminuem(HURD; AMESSE; RANDOLPH, 2005).

$O$ estrogênio também exerce efeito antiarteriosclerótico sobre os vasos sanguíneos. Isso parece resultar, em parte, de um efeito direto sobre os vasos sanguíneos que não é revertido pelo progestogênio. $\mathrm{O}$ estrogênio parece ser um antioxidante que diminui a formação de peroxidases lipidicas, o que pode evitar a arteriosclerose através da minimização da oxidação da lipoproteína de baixa densidade, um potente indutor da formação de placas nos vasos. Um outro efeito benéfico é o da vasodilatação. Parece haver efeito direto sobre as células endoteliais dos vasos sanguíneos que resulta em vasodilatação imediata, talvez mediada por receptores estrogênicos (MARINHO et al., 2005).

A estrogenoterapia em baixas doses (isto é 0,625 $\mathrm{mg} / \mathrm{dia}$ de estrogênios conjugados) resulta em diminuição subclinica da coagulabilidade por redução daagregação plaquetáriae dofibrinogênioe por inibição da formação de plasminogênio. Este efeito parece ser perdido com doses maiores de estrogênio (istoé, 1,25 mg de estrogênios conjugados). Em doses ainda maiores, equivalentes ás usadas em contraceptivos orais, há um aumento da coagulabilidade. Portanto, devem ser usadas doses de estrogênio, equivalentes a 0,625 $\mathrm{mg}$ de estrogênios conjugados para tratamento prolongado (SOCIEDADE BRASILEIRA DE CLIMATÉRIO, 2005).

\section{Vantagens da TRH em relação à Osteoporose}

Há na literatura, evidências definitivas acerca da eficácia da TRH na redução de fraturas por osteoporose (estrogênio isolado ou associado ou associado a progestagênios. O estrogênio conserva o cálcio aumentando a eficiência da absorção intestinal e aumentando a conservação renal de cálcio. Além disso, o estrogênio parece ter efeito direto sobre a função dos osteoclastos. O estrogênio pode reduzir efetivamente a perda óssea e, em grau limitado, pode reverter a perda óssea associada a osteoporose. Por essa razão, a terapia de reposição hormonal deve ser iniciada na menopausa e deve ser administrada por um longo período, embora a duração ideal não seja clara (SOCIEDADE BRASILEIRA DE CLIMATÉRIO, 2005).

$\mathrm{O}$ hipoestrogenismo que se segue à menopausa leva a um aumento da reabsorção óssea, com perda da massa óssea e fraturas de corpos vertebrais, radio distal e colo do fêmur. O estrogênio tem ação direta no osso como droga anti-reabsortiva, e também ações indiretas via paratomônio, vitamina D e calcitonina. Evidências derivadas de estudos clínicos aleatorizados mostram que o uso de estrogênio previne a perda óssea em pacientes saudáveis na pós-menopausa, principalmente se iniciadas logo após a menopausa. Leva também um pequeno ganho de massa óssea, em mulheres na pósmenopausa, particularmente as de coluna vertebral. Existem duvidas em relação à prevenção de fraturas de colo do fêmur (LANZILLOTTI, 2003).

Estudos observacionais mostram que obeneficio tende a desaparecer com a suspensão do tratamento, pois ocorre uma perda acelerada a partir 
de então. Sugerem também que o uso deve ser prolongado, pois o uso por curto prazo não diminui a incidências de fraturas após os 70 anos. O uso de um progestogênio associado cíclico ou continuo não prejudica o beneficio sobre a massa óssea, podendo até ter um efeito adicional. Doses inferiores às habituais também tem efeitos benéficos na preservação da massa óssea (MONTILLA; MARUCCI, 2004).

\section{Fogachos}

O alívio dos fogachos é a razão mais comum que motiva as mulheres a iniciar e continuar a reposição hormonal. Os fogachos podem interferir no ciclo de sono normal e resultam em insônia, e em casos graves, também podem interferir na concentração. Embora a terapia estrogênica oral geralmente seja efetiva na redução da intensidade e da frequiência dos fogachos em poucos dias, as pacientes devem ser advertidas de que podem ser necessárias algumas semanas antes de atingir alivio máximo (HURD; AMESSE; RANDOLPH, 2005).

\section{Efeitos dermatológicos}

O tratamento com estrogênios aumenta a vascularização da pele, levando a alteração no tecido conectivo dérmico. Ocorre um aumento no turn over de ácido hialurônico, incrementando a retenção dérmica de água. A atrofia epidérmica diminui após curto período de estrogenoterapia. Da mesma forma, o decréscimo de colágeno na pele pode ser prevenido ou restaurado. O uso tópico do estradiol aumenta a quantidade de colágeno na pele e tal fato deve-se ao aumento da síntese desta substância e não á redução da sua degradação. O tratamento com estrogênio não aumenta o risco de câncer de pele, embora se saiba que aumenta a fotossensibilidade. A reposição hormonal diminui a queda difusa dos cabelos, pois aumenta a vascularização tanto cutânea, quanto do couro cabeludo, além de prolongar a fase anágena do ciclo e crescimento dos cabelos (SOCIEDADE BRASILEIRA DE CLIMATÉRIO, 2005).

\section{Doença de Alzheimer (DA)}

Já foi demonstrada a presença de receptores para estrogênios em áreas como hipocampo, córtex cerebral, amigdla e loccus ceruleus. Estudos experimentais mostram que o estrogênio estimula o aumento das sinapses e o crescimento neuronal, especialmente dendrítico, reduz também a concentração da substância beta amilóide, aumenta o fluxo sanguíneo cerebral, regula enzimas específicas do cérebro, melhora os níveis de neurotransmissores além de ter um efeito antioxidante (ORCESI et al., 2003).

Estudos aleatorizados controlados, de curta duração, nos quais comparou o estrogênio com placebo, mostram resultados inconsistentes. A metodologia, o tipo de estrogênio, a idade, o tipo de menopausa (natural ou cirúrgica) e principalmente os testes empregados são diferentes. Alguns estudos mostram benefícios em alguns testes, principalmente os voltados para a memória efluência verbal, nas pacientes que utilizaram estrogênios. Uma meta-análise recente conclui que as evidências ainda são pequenas e inconsistentes e não explicadas por melhora dos sintomas e alivio da depressão, indicando necessidade de se avaliar os vários tipos de TRH empregados. As evidências que associavam o uso de estrogênio com a prevenção da doença de Alzheimer são ainda pouco consistentes. Alguns estudos observacionais, casocontrole e coorte mostraram uma redução na incidência de DA em mulheres usuárias de estrogênio comparadas com as não usuárias. Nem todos os estudos, porém mostraram resultados favoráveis (MARINHO et al., 2005).

\section{Tromboembolismo Venenoso e TRH}

O papel dos estrógenos e progestogênos utilizados na reposição hormonal no climatério sobre o sistema cardiovascular são examinados em extensa literatura. Neste sistema, a menopausa tem como aspectos desfavoráveis a elevação da resistência à 
insulina, da resistência vascular, da atividade das enzimas conversoras do angiotensinogênio e da pressão arterial; além disso, ocorre diminuição da liberação de prostaciclina e alteração do perfil lipidico. A própria idade avançada atua negativamente induzindo modificações na estrutura e função cardiovascular. Na parede vascular e no coração, os estrogênios bloqueiam os canais de cálcio, atenuam a vasoconstrição, inibem a proliferação e migração do miócito, reduzem a produção de endotelina e ativam as enzimas prostaciclina e oxido nítrico sintetase. A progesterona inibe as contrações cálcio-independentes das artérias coronárias, mas os progestogênos antagonizam os estrogênios nas ações de inibição da proliferação musculare promoção de vasodilatação (CAMPIOLO; MEDEIROS, 2003).

A incidência de trombose venosa em todas as idades é de 6 a 18 pessoas por 10.000/ano. Essa incidência aumenta com a idade, podendo chegar a 42 casos por 10.000 mulheres/ano nos indivíduos acima de 60 anos. Os estudos indicam que a TRH muda o sistema de homeostasia, reduzindo os inibidores naturais de coagulação e aumentando a atividade fibrinolitica. As complicações tromboembolicas entre as mulheres menopausadas recebendo TRH podem ser secundarias a defeitos adquiridos ou congênitos da coagulação não identificados no inicio da terapia (ORCESI et al., 2003).

\section{Variação da pressão arterial em usuáriasde TRH}

A hipertensão tem sido uma das contraindicações relativas aos contraceptivos orais, pela verificação de que as formulações de maior dosagem aumentam ainda mais a pressão arterial. Em contraste, nas dosagens usadas para terapia de reposição hormonal, os estrogênios conjugados parecem ter pequeno efeito sobre a pressão arterial. Como a hipertensão crônica é um fator de risco bem estabelecido para infarto do miocárdio e acidente vascular cerebral, as mulheres com este distúrbio devem ser incentivadas a manter baixos níveis de pressão arterial e considerar o possível efeito protetor (HURD; AMESSE; RANDOLPH, 2005).

Há muito tempo é relatada a associação entre o uso de hormônios exógenos e hipertensão arterial. Já foi demonstrado que determinados pacientes desenvolveram hipertensão com o uso de estrogênios em anticoncepção oral. Além disso, algumas mulheres na pós-menopausa, reconhecidamente saudáveis, desenvolvem hipertensão arterial ao iniciar TRH, voltando ao padrão anterior de pressão arterial após a interrupção da medicação. Existem evidências de que compostos estrogênicos, naturais ou sintéticos, têm um efeito estimulador no sistema reninaangiotensina II - aldosterona. O efeito básico seria o aumento da produção do substrato da renina pelo fígado, que é então convertido em angiotensina I nos rins e depois em angiotensina II nos pulmões. A angiotensina II, ligando-se aos receptores na superfície das células endoteliais, inicia o processo de vasonstrição, aumentando a pressão arterial e a produção de aldosterona pela adrenal (NAHÂS, 2003).

Considera-se hoje, porém, que a hipertensão desenvolvida por algumas usuárias de terapia de reposição hormonal deve-se ao efeito do progestogênio utilizado em associação ao estrogênio, ou a uma resposta idiossincrática de indivíduos geneticamente propensos. Por outro lado, recentes estudos sugerem que os estrogênios podem ter efeito direto na função contrátil vascular, contribuindo para a vasodilatação, com consequiente diminuição da pressão arterial. Já foi demonstrada a presença de receptores estrogênicos específicos em células endotelias e em células da musculatura lisa de vasos humanos e a regulação da proliferação das células musculares podem ser inibida diretamente pelo estrogênio. Os estrogênios, ligando-se aos receptores endoteliais, aumentam a produção de prostaciclina substancia sabidamente vasodilatadora local de oxido nítrico, outro potente vasodilatador (VIGETA; BRETÃS, 2004). 


\section{TRH e risco de câncer de mama}

Muitos estudos de observação retrospectivos e prospectivos avaliaram se os estrogênios exógenos na forma de contraceptivos orais ou de terapia de reposição estrogênica têm algum impacto sobre a incidência de câncer de mama. Embora algumas metanálises recentes tenham concluído que não há aumento do risco desta neoplasia maligna em mulheres que recebem reposição hormonal, outras demonstraram um aumento significativo do risco de câncer de mama que pode estar relacionado à duração do uso de estrogênio. Um destes estudos mostrou um risco relativo de desenvolvimento de câncer de mama de 1,46 em mulheres que tomaram estrogênios após a menopausa por cinco anos ou mais. Entretanto, ainda não foram concluídos grandes estudos clínicos prospectivos e os benefícios da reposição hormonal podem superar este possível risco em algumas pacientes (HURD; AMESSE; RANDOLPH, 2005).

Marinho et al.(2005) basearam-se nos estudos existentes, em relação ao risco de câncer de mama em mulheres que fazem uso da estrogenioterapia ou da estroprogestagenioterapia e puderam verificar que:

a) estrogenoterapia de curta duração (uso menor do que cinco anos): não há aumento do risco de câncer de mama.

b) estrogenoterapia em longo prazo (uso igual ou maior que cinco anos): há um pequeno aumento do risco, o qual parece ainda aumentar com o tipo de uso (usuárias atuais) e com idade (acima de 55 anos).

c) a estroprogestagenioterapia, em face das publicações mais recentes e de maior porte, parece provocar aumento maior do risco de câncer de mama, principalmenteemesquemas cíclicos, sendoproporcional ao uso atual ou recente e ao tempo de uso.

d) antes que a resposta definitiva ao risco de câncer de mama em mulheres que utilizam os dois tipos de TRH esteja disponível, por meio dos ensaios clínicos aleatórios, seria prematuro não recomendála sistematicamente às mulheres pós-menopaúsicas com risco importante para doença cardiovascular e/ ou osteoporose, não é recomendado basear-se apenas nos riscos oriundos dos estudos epidemiológicos atualmente conhecidos, ao contrário saber sempre individualizar a sua utilização.

e) A relação risco/beneficio deve ser cuidadosamente verificada em mulheres, principalmente as de mais idade, que irão começar a fazer o uso de TRH por tempo prolongado. Neste particular, principalmente nas mulheres com fatores de risco para câncer de mama, seria prudente indicar alguma forma de terapia hormonal que utilize, em mulheres com útero progestogênios com intervalos de tempo maiores em esquemas cíclicos (com preferência para uso a cada 3 ou 4 meses) ou em esquemas combinados- contínuos. Após cinco anos de uso, quando começa a aumentar o risco de tumor de mama, podem ser utilizadas substâncias que tenham ação inibitória sobre a proliferação do epitélio mamário e endometrial e possibilitem boa parte dos benefícios estrogênicos sobre outros tecido efetores.

\section{TRH e risco de câncer no endométrio}

Um risco bem estabelecido da terapia de reposição estrogênica é o de hiperplasia e câncer do endométrio. Estudos de observação iniciais verificaram quemulheres que usaram apenas estrogênio eram quatro a sete vezes mais propensas a desenvolver câncer do endométrio que as outras. O uso simultâneo de progestogênios previne efetivamente este problema na maioria dos casos. Entretanto, deve ser lembrado que mesmo mulheres em tratamento com progestogênio podem desenvolver câncer do endométrio, principalmente se usaram estrogênios sem oposição no passado. Portanto, a avaliação do endométrio ainda é uma parte importante do tratamento de todas as mulheres com sangramento vaginal irregular (ALDRIGHI; FALUDI; MANSUR, 2005).

Oendométrioproliferativoécaracterísticoda primeira fase dos ciclos ovulatórios e de qualquer momento de ciclos anovulatórios ou de endométrios acíclicos, desde que submetidos a estimulo estrogênico. A espessura do endométrio proliferativo varia de 2 a $5 \mathrm{~mm}$ na fase 
proliferativa inicial sendo em média de 9 a $11 \mathrm{~mm}$ na tardia. A ação da progesterona acerca do endométrio exige prévio estimulo estrogênico proliferativo, traduzindo-se pelo aspecto serrilhado e tortuoso das glândulas, que contém vacúolos ricos em glicogênioe lipídios, e edema do estroma. A ausência de estimulo progestacional com persistência do estrogênio favorece uma proliferação mais intensa (SOCIEDADE BRASILEIRA DECLIMATÉRIO, 2005).

A exposição contínua do endométrio ao estrogênio, sem a oposição da progesterona (o que ocorre nos ciclos anovulatórios ou na estrogenoterapia), redunda, em período que varia na dependência dos níveis de produção estrogênica ovariana e da maior ou menor presença de receptores estrogênicos nas células endometriais, em proliferação anormal. Esta se caracteriza pela hiperplasia do endométrio, traduzida pelo aumento do número e dimensão das glândulas e do estroma. Muito mais importante que os aspectos glandularestromais da hiperplasia (simples ou complexa), o diagnóstico histológico crucial é a presença ou ausência de atipias celulares, que caracteriza o alto risco para o desenvolvimento do carcinoma de endométrio (ORCESI et al., 2003).

O carcinoma do endométrio é a segunda mais frequiente neoplasia ginecológica no Brasil e a quinta causa de câncer em mulheres. O risco de mulheres desenvolverem esta doença após os 50 naos é de 2 a $3 \%$ e cerca de $90 \%$ dos casos desenvolvem-se após a menopausa. Fatores constitucionais, metabólicos e hormonais encontram-se na gênese do carcinoma do endométrio, devendo o ginecologista estar atento para os principais fatores de risco em sua maioria relacionados com a maior exposição aos estrogênios sem oposição progestacional: anovulação crônica, nuliparidade; menarca precoce; menopausa tardia; estrogenioterapia sem adição de progestagênios; obesidade; diabetes; hipertensão; portadoras de sangramento endometrial anormal e/ou hiperplasia endometrial; historia familiar de câncer de endométrio e ou de mama (SOCIEDADE BRASILEIRA DE CLIMATÉRIO, 2005).
Segundo Fernandes, Baracat e Lima (2005), quatro situações merecem atenção especial do clínico e exigem particular vigilância endometrial seja na peri ou na pós-menopausa. A primeira é a terapêutica de reposição hormonal sem progestagenioterapia associada. O sangramento endometrial anormal, é o principal, e geralmente o primeiro sintoma, do câncer do endométrio, estando presente, embora quase sempre tardiamente, em cerca de $80 \%$ dos casos. A obesidade, associada ou não a diabetes e hipertensão, têm comprovada influência na gênese dessa neoplasia. Finalmente, a hiperplasia de endométrio é fator altamente predisponente, havendo consenso entre a maioria dos autores que a hiperplasia do endométrio com atipias é lesão precursora, ocorrendo transformação maligna em 25 a $40 \%$ dos casos. Saliente-se que a hiperplasia sem atipias responde bem (cerca de 95\% dos casos) a progestagenioterapia.

De acordo com a SOCIEDADE BRASILEIRA DE CLIMATÉRIO (2005), o adenocarcinoma do endométrio estrogênio-dependente é o mais freqüente dos tipos de câncer do endométrio, e apresenta as seguintes características: associa-se à hiperplasia; é bem diferenciado; invade tardiamente o endométrio. Essas características propiciam bom prognóstico e ressaltam a importância da vigilância endometrial na peri e pós- menopausa que pode ser resumida nos seguintes objetivos:

a) avaliação clinica dos fatores de risco.

b) propedêutica eficaz no diagnostico das lesões precursoras.

c) avaliação prévia e no seguimento da terapêutica de reposição hormonal que enseje uma menor incidência de efeitos colaterais e em conseqüência, maior adesão das usuárias ao tratamento. O carcinoma tipo II não é precedido por estas lesões. È tardio, de mau prognostico e quase sempre do tipo seroso papilífero.

Acompanhamento da Paciente durante a terapia hormonal

Cumpre ao clínico investigar as alterações que ocorrem no climatério, bem como o rastreamento 
de neoplasias, avaliação do risco cardiovascular, diagnóstico de endocrinopatias concomitantes e avaliação da função hepática, hematológica e renal, além de avaliar as pacientes com potencial para desenvolver osteoporose antes do inicio da terapêutica. Em geral, o quadro clínico atesta a eficiência do esquema terapêutico utilizado para assegurar que a dose oferecida é adequada, isto é, acima de $40 \mathrm{pg} / \mathrm{ml}$. Portanto, a melhora dos sintomas vasomotores e neuropsíquicos sugere, via de regra que a dosagem de estrogênio é suficiente. Além disso, durante o exame ginecológico, é possível aquilatar o grau de estrogenização pela coloração rósea da vagina e do colo, e pela presença de muco cervical com certa filância e cristalização. Além disso a estrogenização também pode ser avaliada pelo esfregaço vaginal, que era anteriormente atrófico (com predomínio de células basais) e torna-se estrogênico (com poucas células basais e parabasais e maior quantidade de células intermediarias e superficiais). Assim, pode-se atestar a eficácia da dose de estrogênio empregada. Ainda na monitoração da eficácia da TRH, deve-se avaliar cuidadosamente o grau de trofismo da pele e das mucosas em geral (HANG, 2001).

Segundo Fernandes, Baracat e Lima (2004) os principais exames complementares na rotina do climatério são:

a) Rastreamento de neoplasias-mamografia e ultra-sonografia mamária, ultra-sonografia pélvica transabdominal e/ou transvaginal, ultra-sonografia pélvica transabdominal e/ou transvaginal, histeroscopia com biopsia endometrial dirigida ou curetagem uterina, citologia cervicovaginal oncologica e colposcopia, sangue oculto nas fezes

b) Avaliação do risco de doença cardiovascularcolesterol total e frações, triglicérides, glicemia de jejum, eletrocardiograma.

c) Avaliação do trato urogenital: urina I e citologia urinária, estudo urodinâmico, índice de maturação celular. d) Avaliação da função hepática: dosagem de albumina ou proteinograma, TGO, TGP, bilirrubinas totais e frações

e) Avaliação da função renal - uréia, creatinina

f) Avaliação hematológica da função da medula óssea: hemograma completo

g) Avaliação da remodelação óssea: densitometria óssea, marcadores bioquímicos de formação e reabsorção óssea.

h) Avaliação da função tireoidiana: TSHe T4 livre.

\section{Interações medicamentosas dos estrogênios}

De acordo com Polden e Mantle (1998) a rifampicina comprovadamente diminui a atividade dos estrogênios quando administrada concomitantemente. Esse efeito tem sido atribuído ao aumento da metabolização de estrogênio, provavelmente por indução de enzimas microssomais hepáticas. As seguintes interações medicamentosas foram relatadas quando em emprego simultâneo com contraceptivos orais:

a) Interferência na eficácia de alguns agentes anti-hipertensivos, anticoagulantes orais, anticonvulsivantes e hipoglicemiantes.

b) Alteração na eficácia de outras drogas, como teofilina, fenotiazinas, corticosteróides, antagonistas beta-adrenérgicos, antidepressivos tricíclicos, cafeína e ciclosporina, sejam por potencialização/aumento dos efeitos farmacológicos ou pela sua diminuição.

c) Interferência no metabolismo oxidativo do diazepan e clordizepóxido, provocando seu acumulo plasmático. Pacientes utilizando tais drogas ou em tratamento prolongado com benzodiazepinícos devem ser monitoradas quanto á possível diminuição dos seus efeitos ou intensificação dos efeitos sedativos. Os efeitos dos benzodiazepinícos no metabolismo de contraceptivos orais ainda não foram determinados. 
Avaliação da Aceitabilidade da Terapia de

\section{Reposição Hormonal}

No que concerne ao benefício máximo da reposição hormonal no climatério, sabe-se que ele se relaciona com o uso contínuo e com a adesão ao tratamento. A cooperação e a orientação da paciente são requisitos básicos para o sucesso da prevenção dos efeitos deletérios do hipoestrogenismo. No entanto, é importante mencionar que apenas $20 \%$ das mulheres prosseguem com o uso da terapia hormonal no climatério. As demais a abandonam em decorrência dos efeitos colaterais e do receio de desenvolverem câncer. Entre os principais fatores que modulam a aceitabilidade da hormonioterapia dos progestogênios que simulam a síndrome pré-mestrual e o receio de desenvolvimento de carcinoma de mama e de endométrio. Recentemente, após a divulgação pela mídia dos resultados do Women's Health Iniatiate, muitas mulheres estão mais preocupadas e ansiosas por conta da possibilidade de desenvolverem câncer mamário e doenças cardiovasculares com o uso de terapia hormonal (VIGETA; BRÊTAS, 2004).

Durante o acompanhamento clínico da terapia hormonal, pode-se deparar com efeitos colaterais provenientes da ação estrogênica e/ou progestacional. Por isso, as pacientes devem ser bem esclarecidas a esse respeito. Assinalam-se entre, os principais efeitos adversos dos estrogênios, a retenção hidrossalina (aumento de peso, edema, mastalgia), a cefaléia e a metrorragia (proliferação endometrial). Devem-se, pois, utilizar medidas para combatê-las, como associar diurético quando houver indícios de retenção hídrica importante, analgésicos para a cefaléia e a mastalgia. Se não houver respostas satisfatórias, é possível mudar a via de administração ou o tipo de estrogênio. Já o uso de progestogênios costuma determinar dispareunia, pois a vagina continua ou torna-se mais seca. Podem ocorrer também sintomas semelhantes aos da síndrome pré-menstrual, em especial instabilidade emocional, irritabilidade, depressão cíclica e dor mamária. Se houver história prévia de depressão pré-menstrual ou de depressão mascarada, deve-se evitar os progestogênios de ação prolongada (BARACAT; LIMA , 2005).

\section{Conclusão}

Uma das reconhecidas vantagens da terapêutica hormonal éo alívio dos sintomas vasomotores, atrofia gênito-urinária e prevenção da osteoporose. $\mathrm{O}$ uso dessa terapia deve ser recomendada pelo médico de comum acordo com a paciente e exige um acompanhamento adequado da mesma. Quando recomendada à terapêutica hormonal deve ser utilizada o tempo que se julgue necessário na menor dose eficaz. Os esquemas terapêuticos, os hormônios a serem utilizados e a via de administração, devem ser adaptados às necessidades específicas de cada paciente. A terapia estrogênica isolada, ao contrário da terapia estroprogestativa, não parece aumentar o risco relativo de câncer de mama e doença coronariana.

Quando houver sintoma de hipoestrogenismo, existe indicação clara para o uso da TRH, pelo menos por curto tempo. A TRH não deve ser prescrita se houver uma contra-indicação ou uma decisão da paciente em não usar, desde que adequadamente informada. O uso prolongado para a prevenção de doenças degenerativas, especialmente em mulheres assintomáticas, deve ser decidido individualmente, de acordo com as características e risco de cada mulher. A decisão dependerá do risco para cada doença sobre a qual a TRH possa interferir da compreensão da paciente em relação às evidências da literatura e do desejo e disposição em fazer o uso prolongado da medicação.

Sendo assim, todo médico, antes de prescrever a TRH, deve ter um diálogo franco com suas pacientes, informando sobre o significado e as conseqüências da deprivação estrogênica no climatério e os riscos, benefícios, efeitos colaterais e contra-indicações da mesma. Por isso, é importante considerar que só a sabedoria adicional, proveniente de pesquisas em andamento, permitirá 
maior aperfeiçoamento da capacidade de prevenir e tratar doenças nas mulheres após a menopausa. Portanto, nunca deve ser esquecido o papel fundamental exercido pela atividade física, dieta equilibrada e abolição do tabagismo na prevenção de doenças. Ainda é importante a realização de mamografia, bem como ecografia pélvica transvaginal além da monitorizarão da pressão arterial, da glicose, do colesterol e dos triglicérideos séricos antes de iniciar qualquer TRH.

O farmacêutico será o último contato que a paciente climatérica, terá com um profissional de saúde antes de iniciar o seu tratamento com a TRH. Sendo assim é importante que este, acrescente informações adicionais, complementando as orientações do médico, a respeito do ínicio do tratamento, bem como precauções, interações medicamentosas, reações adversas e horários de administração, otimizando o tratamento da paciente, fazendo com que a mesma cesse todas as suas dúvidas a respeito da TRH.

\section{Referências}

ALDRIGHI, J. M.; FALUDI, A. A.; MANSUR, A. de P. Doença cardiovascular no climatério. In: ALDRINGHI, J. M.; CARDOSO JUNIOR, R. Climatério e menopausa. São Paulo: Atheneu, 2005.

BARACAT, E. C.; HAIDAR, M. A.; NUNES, M. G; SOARES JUNIOR, J. M.; LIMA, G. R. Guia de medicina ambulatorial e hospitalar. In: BARACAT, E. C.; LIMA, G. R. Climatério. São Paulo: Manole, 2005.

BARACAT, E C.; LIMA, G. R. Ultrasonografia transvaginal na pós-menopausa. São Paulo: Manole, 2005.

CAMPIOLO, D. J.; MEDEIROS, S. Tromboembolismo venoso e terapia de reposição hormonal da menopausa: uma análise clinicoepidemiológica. Revisão de Literatura. Universidade de Cuiabáe Universidade Federal de Mato Grosso. Departamento de Ginecologia e Obstetrícia, v.47, n.5, p.534-541, out. 2003.

CHUERY, A. C. S.; BAGNOLI, V. R.; FONSECA, A. M.; PARELLADA, C. I. Hormonioterapia na menopausa. Disponível em: $<$ http/www.portaldeginecologia.com.br $>$ Acesso em: 20 jan. 2005.

CLAUPAUCH, R.; MEIRELLES, R. M. R.; JULIÃO, M. A.; LOUREIRO, C. K.; GIARODOLI, P. B; PINHEIRO, S. A.; HARRIGAN, A. R.; SPRITZER, P. M.; PARDINI, D. P.; WEISS, R. V.; ATHAYDE, A.; RUSSO, L. A.; POVOA, L. C. Fitoestrogênios: Posicionamento do Departamento de Endocrinologia Feminina da Sociedade Brasileira de Endocrinologia e Metabologia. Arquivos Brasileiros de Endocrinologia e Metabologia, São Paulo, v.26 n.6, p 679-691, nov./dez. 2002.

DOUGLAS, C. R. Tratado de fisiologia aplicada a saúde. In: BYDLOWSKI, S. P. Fisiologia da gônada feminina. São Paulo: Robe Editorial, 2002. p.

FERREIRA, J. A. de S. Climatério feminino (Fisiopatologia, diagnóstico e tratamento). In: FERNANDES, C. E.; MELO, N. R.; WEHBA, S. A perimenopausa. São Paulo: Lemos Editorial, 1999.

HAIDAR, C.; BOUNDUKI, M.; BARACAT, E. Climatério feminino (Fisiopatologia, diagnósticoe tratamento). In: FERNANDES, C. E.; MELO, N. R.; WEHBA, S. Indicações e contra indicações na reposição hormonal em mulheres na pósmenopausa. São Paulo: Lemos Editorial, 1999. p.

FERNANDES, C. E.; BARACAT, E. C.; LIMA, G. R. Climatério: manual de orientação. São Paulo: Ponto, 2004. Disponível em: < http/ www.febrasgo.org.br>. Acesso em 20 jan. 2005.

FRANÇA, A. P.; ALDRIGHI, J. M.; MARUCCI, M. de F. N. Doença cardiovascular 
no climatério. In: ALDRINGHI, J. M.; CARDOSO JUNIOR, R.. Terapia de reposição hormonal da doença cardiovascular: fioestrogênios. São Paulo: Atheneu, 2005. p.

GONÇALVES, W. J.; BARACAT, E. C.; BORTOLLETTO, C. de C. R.; HAIDAR, M. A.; LIMA, G. R._Guia de medicina ambulatorial e hospitalar. In: BARACAT, E. C.; LIMA, G. R.. Ultra-sonografia Transvaginal na pósmenopausa. São Paulo: Manole, 2005. p.

HARDY, E.; OSIS, M. J. D.; MARTINEZ, E. Z. Caracterização das usuárias da terapia de reposição hormonal de Campinas, São Paulo: Revisão de literatura. Universidade Estadual de Campinas. Departamento de Toxoginecologia, v.1 n.1, p.121-126, jan./fev. 2002.

HURD, W. W; AMESSE, L. S; RANDOLPH, J. F. Tratado de ginecologia. In: BEREK, J. S.; ADASHI, E.; HILLARD. Menopausa. Rio de Janeiro: Guanabara Koogan, 2005. p.

LANZILLOTTI, H. S.; LANZILLOTTI, R. S.; TROTTE, A. S. D.; DIAS, A. S.; BORNAND, B.; COSTA, E. A. M. M.. Osteoporose em mulheres na pós-menopausa, cálcio dietético e outros fatores de riscos. Universidade do Estado do Rio de Janeiro Departamento de Nutrição, v.1 n.1, p.182-191, abr./jun. 2003.

MARINHO, R. M.; FERNANDES, C.E.; WEHBA, S; PINTONETO, A. M; BARACAT,E.C. Projeto diretrizes: atenção primária e terapia de reposição hormonal no climatério. Disponívelem: <http/ www.febrasgo.org.br>. Acesso em: 20 jan. 2005.

MONTILLA, R. das N. G.; MARUCCI, M. de F. N. Relação cálcio/proteína da dieta de mulheres climatérias: Revista Associação Médica Brasileira Universidade de São Paulo, v.1 n.1,p.52-54, out./nov. 2004.
NAHÂS, E. A. P.; TRAIMAN , P.; PONTES, A.; DALBEN, I. Efeitos da Isoflavona sobre os sintomas climatérios e o perfil lípidico na mulher em menopausa. Universidade Estadual de São Paulo Departamento de Ginecologia e Obstetrícia, v.25 n.5, p.337-342, 2003.

ORCESI, A.; MENDES NETO, A.; SIMÔES, L. H.; OSIS, M. J. D.; HARDY, E. E. Síndrome do climatério: Inquérito populacional domiciliar em Campinas, SP. Revista Saúde publica. Universidade de Campinas Departamento de Tocoginecologia, p.735-740, 2003. Disponível em: < http/www.fsp.usp.br>. Acesso em: 20 jan.2005.

PIATO, S. Tratado de ginecologia. In: FONSECA, A. M.. Terapia de reposição hormonal. São Paulo: Artes Médicas, 1997.p.

POLDEN, M.; MANTLE, J. Fisioterapia em ginecologia e obstetrícia. In: Oclimatério. São Paulo: Santos, 1998. p.

RANG, H. P.; DALLE, M. M.; RITTER, J. M. Farmacologia aplicada. In: Hormônios gonadotropicos. São Paulo: Guanabara Koogan, 2001. p.

SANTOS, L. de O. M.; PESSOLE, M. de L.; IOSHII, S. O. Efeito dos estrógenos conjugados e da Medroxiprogesterona sobre a mama. Revista brasileira de ginecologia e obstetrícia, v.23 n.8, p.23-35, set./out. 2001. Disponível em: <http/ www.scielo.br>. Acesso em 20 jan. 2005.

SOCIEDADEBRASILEIRADECLIMATÉRIO (SOBRAC). Consenso brasileiro multidisciplinar de_assistência á saúde da mulher climatéria. Disponível em: $\langle\mathrm{http} / \mathrm{www}$.sobrac.org.br >. Acesso em: 20 jan. 2005.

SOCIEDADEBRASILEIRA DECLIMATÉRIO (SOBRAC). Consenso da Sobrac: "Terapêutica hormonal na peri e na pós-menopausa". Disponível 
em: <http/www.sobrac.org.br>. Acesso em: 20 jan. 2005.

STANCEL, G. M; MITCHELL, D. L.. As bases farmacológicas da terapêutica. In: GILMAN, A. G. Estrogênios e progestogênios. São Paulo: Guanabara Koogan, 2003.

VIGETA, S. M. G.; BRETÃ S, A. C. P. A experiência da perimenopausa e pós-menopausa com mulheres que fazem o uso ou não da terapia de reposição hormonal: Universidade Federal de São Paulo Departamento de Enfermagem, v.1 n.1, p.1682-1688, nov./dez. 2004.

WALL, R. E; DONA, M.D; OKUDA, M.D. Segredos em ginecologia e obstetrícia. In: FREDERICKSON, H. L.; HAUG, W. L. Menopausa. São Paulo: Artes Médicas Sul, 2003.p. 